\title{
Kaonic deuterium from realistic antikaon-nucleon interaction
}

\author{
Wataru Horiuchi ${ }^{1}$, Tetsuo Hyodo ${ }^{2, *}$, and Wolfram Weise ${ }^{2,3}$ \\ ${ }^{1}$ Department of Physics, Hokkaido University, Sapporo 060-0810, Japan \\ ${ }^{2}$ Yukawa Institute for Theoretical Physics, Kyoto University, Kyoto 606-8502, Japan \\ ${ }^{3}$ Physik-Department, Technische Universität München, 85748 Garching, Germany
}

\begin{abstract}
We present precise three-body calculations for the spectrum of kaonic deuterium with a realistic antikaon-nucleon interaction. Thanks to the precise measurement of kaonic hydrogen, it is now possible to construct realistic $\bar{K} N$ interactions which reproduce the whole set of experimental data in the threshold region. Employing such realistic interactions, the energy of the threebody system of kaonic deuterium is determined with the accuracy of $\mathrm{eV}$, by expanding its wave function with a large number of correlated Gaussian basis functions. The level shift and width of the $1 S$ state are found to be $670 \mathrm{eV}$ and $1016 \mathrm{eV}$, respectively. The improved Deser formulas work reasonably well to estimate the shift and width of kaonic hydrogen, but their application to kaonic deuterium does not give an accurate estimation. It is shown that the result is sensitive to the $I=1$ component of the $\bar{K} N$ interaction, which will be further constrained in future experiments.
\end{abstract}

\section{Introduction}

The antikaon-nucleon $(\bar{K} N)$ interaction is one of the intensively studied subjects in the strangeness nuclear physics [1]. This two-body interaction is closely related with the properties of the $\Lambda(1405)$ resonance which exists near the $\bar{K} N$ threshold [2-6]. In addition, the determination of the $\bar{K} N$ interaction is crucial for the prediction of antikaon bound systems in nuclei, called kaonic nuclei $[7,8]$. The precise measurement of kaonic hydrogen by the SIDDHARTA collaboration $[9,10]$ is regarded as one step forward to determine the $\bar{K} N$ interaction, because the uncertainty of the $\bar{K} N$ interaction, in particular in the subthreshold energy region, is significantly reduced by this data $[11,12]$. The properties of the $\Lambda(1405)$ shown in the particle listing in PDG [13] have been updated according to the comprehensive analyses of the $\bar{K} N$ scattering data $[11,12,14,15]$. While the isospin $I=0$ component of the $\bar{K} N$ interaction is well constrained by the SIDDHARTA results together with the $K^{-} p$ scattering data, there still remains the uncertainty in the $I=1$ component $[6,11,12]$.

Kaonic deuterium is basically a hydrogen-like system of the $K^{-}$and the deuteron bounded by the Coulomb interaction. The strong interaction between the $K^{-}$and the nucleons, however, shifts the energy of the lowest $1 S$ state from the purely electromagnetic value, and induces the finite decay width due to the absorption to the lower energy $\pi \Sigma$ and $\pi \Lambda$ systems. Because kaonic deuterium contains $K^{-} n$ pair which is purely in $I=1$, this system is

\footnotetext{
*e-mail: hyodo@yukawa.kyoto-u.ac.jp
} 
considered to provide further constraints on the isospin dependence of the $\bar{K} N$ interaction, complementary to the kaonic hydrogen data. In fact, new experiments to measure kaonic deuterium are planned: J-PARC E57 [16] and SIDDHARTA-2 [17, 18].

Here we show the theoretical prediction of the energy levels of kaonic deuterium in Ref. [19]. In contrast to previous works, we have combined

(i) the rigorous three-body treatment, and

(ii) the realistic $\bar{K} N$ interaction constrained by the SIDDHARTA data.

These are the essential requirements for reliable predictions of kaonic deuterium (see also Faddeev approaches in Refs. [20, 21]). In order to accurately describe the three-body system with both strong and electromagnetic interactions, a large number of correlated Gaussian basis functions are used to expand the wave function [22-25], so that the requirement (i) is fulfilled. The Kyoto $\overline{K N}$ potential in Ref. [26] is employed to satisfy the requirement (ii). In the following, we briefly introduce the construction of such realistic $\bar{K} N$ potentials, and then apply them to calculate kaonic atoms.

\section{Realistic $\bar{K} N$ potentials}

Regarding the antikaon as a Nambu-Goldstone boson associated with the spontaneous breaking of three-flavor chiral symmetry, we can describe the low-energy $\bar{K} N$ interaction in the framework of chiral SU(3) dynamics [2-6]. Around the $K^{-} p$ threshold, there exists the following set of experimental data:

- $K^{-} p$ total cross sections,

- threshold branching ratios, and

- level shift $\Delta E_{K^{-}} p$ and width $\Gamma_{K^{-}}$of kaonic hydrogen.

It is shown in Refs. $[11,12]$ that all these data can be well described by the next-to-leadingorder (NLO) chiral SU(3) dynamics with the high accuracy of $\chi^{2} /$ d.o.f. $\sim 1$. Among others, the kaonic hydrogen measurement by the SIDDHARTA collaboration $[9,10]$ provides a crucial constraint on the meson-baryon scattering amplitude.

Although the satisfactory scattering amplitude is obtained, the field theoretical framework in Refs. $[11,12]$ is not directly applicable to few-body calculations. For the application to kaonic deuterium, it is useful to construct a local potential in the coordinate space, which equivalently reproduces the results of chiral SU(3) dynamics. The basic strategy to construct such an equivalent potential has been developed in Ref. [27]. It is shown that the scattering amplitude of chiral SU(3) dynamics can be equivalently described by the local potential with an energy-dependent potential strength, plugged in the Schrödinger equation.

The $\bar{K} N$ potentials equivalent to the up-to-date NLO chiral SU(3) amplitude are constructed for the single-channel $\bar{K} N$ system [26] and for the coupled-channels $\bar{K} N-\pi \Sigma-\pi \Lambda$ system [28]. In this way, the realistic $\bar{K} N$ potentials, which reproduce the whole set of experimental data around the $K^{-} p$ threshold with $\chi^{2} /$ d.o.f. $\sim 1$, are now available. For the application to kaonic atoms, we use the single-channel version called Kyoto $\bar{K} N$ potential [26], where the effect of the lower-energy $\pi \Sigma$ and $\pi \Lambda$ channels are incorporated in the imaginary part of the potential strength.

\section{Application to kaonic atoms}

Now we apply the Kyoto $\bar{K} N$ potential for the calculation of kaonic hydrogen and kaonic deuterium. We solve the Schrödinger equation using a variational method with basis expansion. 
This is computationally challenging even for the three-body system of kaonic deuterium, because we have to treat the long-range Coulomb interactions and the short-range strong interactions simultaneously. In the coordinate space, this corresponds to cover the distance scales from $0.1 \mathrm{fm}$ to several hundreds of fm. Introducing a large number of correlated Gaussian basis functions [22-25] to express the spatial part of the wave function, the energy convergence is achieved at $\mathrm{eV}$ level in the calculation of kaonic deuterium. More detailed procedures are explained in Ref. [19].

The energy of kaonic atoms is frequently estimated by the improved Deser formula, which was derived by incorporating the isospin symmetry breaking effect with the effective field theory technique $[29,30]$. The $1 S$ level shift $\Delta E$ and width $\Gamma$ of kaonic hydrogen (kaonic deuterium) can be estimated by

$$
\Delta E-\frac{i \Gamma}{2}=-2 \mu^{2} \alpha^{3} a[1-2 \mu \alpha(\ln \alpha-1) a]
$$

where $\mu$ is the $K^{-} p\left(K^{-} d\right)$ reduced mass, $\alpha$ is the fine structure constant and $a$ is the $K^{-} p$ $\left(K^{-} d\right)$ scattering length. Further improved formula has been proposed in Ref. [31] by resumming the logarithmically enhanced correction term as

$$
\Delta E-\frac{i \Gamma}{2}=-\frac{2 \mu^{2} \alpha^{3} a}{1+2 \mu \alpha(\ln \alpha-1) a} .
$$

We will examine the validity of these formulas in comparison with the exact calculations.

\subsection{Kaonic hydrogen}

We first calculate kaonic hydrogen. Although the kaonic hydrogen data is used to constrain the original scattering amplitude in Refs. [11, 12], it is legitimate to perform this calculation with the Kyoto $\bar{K} N$ potential because of the following reasons;

- In Refs. [11, 12], the improved Deser formula (1) is used to derive the constraint on the scattering amplitude from the kaonic hydrogen result.

- Physical hadron masses with isospin symmetry breaking are used in the meson-baryon amplitude of Refs. [11, 12], but the Kyoto $\bar{K} N$ potential [26] is formulated with the isospin averaged masses.

While the isospin symmetry breaking effect can be safely neglected for the calculation of kaonic nuclei which has the binding energy of several tens of $\mathrm{MeV}$, in the energy scale of kaonic atoms, the mass difference between the $K^{-} p$ and $\bar{K}^{0} n$ cannot be neglected. Thus, we perform the two-body calculation with the following coupled-channel Schrödinger equation

$$
\left(\begin{array}{cc}
\hat{T}+\hat{V}^{\bar{K} N}+\hat{V}^{\mathrm{C}} & \hat{V}^{\bar{K} N} \\
\hat{V}^{\bar{K} N} & \hat{T}+\hat{V}^{\bar{K} N}+\Delta m
\end{array}\right)\left(\begin{array}{l}
\left|K^{-} p\right\rangle \\
\left|\bar{K}^{0} n\right\rangle
\end{array}\right)=E\left(\begin{array}{c}
\left|K^{-} p\right\rangle \\
\left|\bar{K}^{0} n\right\rangle
\end{array}\right),
$$

where $\hat{T}$ is the kinetic energy operator, $\hat{V}^{\bar{K}} N$ is the Kyoto $\bar{K} N$ potential, $\hat{V}^{C}$ is the Coulomb interaction, and $\Delta m$ is the mass difference of the $K^{-} p$ and $\bar{K}^{0} n$ channels. Because the strong $\bar{K} N$ interaction causes the transition from $K^{-} p$ to $\bar{K}^{0} n$, it is necessary to include the $\bar{K}^{0} n$ channel.

The energy shift $\Delta E_{K^{-} p}$ and width $\Gamma_{K^{-} p}$ of kaonic hydrogen are obtained as

$$
\left(\Delta E_{K^{-} p}, \Gamma_{K^{-} p}\right)=(283,607) \mathrm{eV}
$$


Table 1. Level shift and width of kaonic hydrogen obtained by solving the Schrödinger equation with the Kyoto $\bar{K} N$ potential, and by using the improved Deser formula (1) and its resummed version (2).

The numerical data are taken from Ref. [19].

\begin{tabular}{lcc}
\hline & $\Delta E_{K^{-} p}(\mathrm{eV})$ & $\Gamma_{K^{-}}(\mathrm{eV})$ \\
\hline Full Schrödinger equation & 283 & 607 \\
Improved Deser formula (1) & 293 & 596 \\
Resummed formula (2) & 284 & 605 \\
\hline
\end{tabular}

which is consistent with the SIDDHARTA result, $\Delta E_{K^{-} p}=283 \pm 36 \pm 6 \mathrm{eV}$ and $\Gamma_{K^{-}} p=541 \pm$ $89 \pm 22 \mathrm{eV}[9,10]$. This verifies the use of the Kyoto $\bar{K} N$ potential together with the physical hadron masses for the calculation of kaonic atoms. In this setup, the $K^{-} p$ scattering length is calculated as $a_{K^{-}} p=-0.66+i 0.89 \mathrm{fm}$. The estimation of $\left(\Delta E_{K^{-}}, \Gamma_{K^{-}}\right)$by the improved Deser formulas are shown in Table 1. We see that the exact solution from the Schrödinger equation is well estimated by these formulas. In particular, the resummed version gives a good estimation which has only a few $\mathrm{eV}$ deviation from the exact value.

\subsection{Kaonic deuterium}

Next, we consider kaonic deuterium. The Schrödinger equation is given by

$$
\left(\begin{array}{cc}
\hat{H}_{K^{-} p n} & \hat{V}_{12}^{\bar{K} N}+\hat{V}_{13}^{\bar{K} N} \\
\hat{V}_{12}^{\bar{K} N}+\hat{V}_{13}^{\bar{K} N} & \hat{H}_{\bar{K}^{0} n n}
\end{array}\right)\left(\begin{array}{l}
\left|K^{-} p n\right\rangle \\
\left|\bar{K}^{0} n n\right\rangle
\end{array}\right)=E\left(\begin{array}{l}
\left|K^{-} p n\right\rangle \\
\left|\bar{K}^{0} n n\right\rangle
\end{array}\right),
$$

with

$$
\begin{aligned}
& \hat{H}_{K^{-} p n}=\sum_{i=1}^{3} \hat{T}_{i}-\hat{T}_{\mathrm{cm}}+\hat{V}_{23}^{N N}+\sum_{i=2}^{3}\left(\hat{V}_{1 i}^{\bar{K} N}+\hat{V}_{1 i}^{\mathrm{EM}}\right), \\
& \hat{H}_{\bar{K}^{0} n n}=\sum_{i=1}^{3} \hat{T}_{i}-\hat{T}_{\mathrm{cm}}+\hat{V}_{23}^{N N}+\sum_{i=2}^{3} \hat{V}_{1 i}^{\bar{K} N}+\Delta M,
\end{aligned}
$$

where the index 1 is assigned to the antikaon and (2,3) to the nucleons, and $\Delta M$ denotes the mass difference of the $K^{-} p n$ and $\bar{K}^{0} n n$ channels. For the nuclear force $\hat{V}_{23}^{N N}$, we adopt the Minnesota force [32].

The energy shift $\Delta E_{K^{-} d}$ and width $\Gamma_{K^{-} d}$ are obtained as

$$
\left(\Delta E_{K^{-} d}, \Gamma_{K^{-} d}\right)=(670,1016) \mathrm{eV} .
$$

It is explicitly demonstrated that the shift in the $2 P$ state is less than $\mathrm{eV}$ level. This validates the determination of the $1 S$ level shift from the transition x-ray from $2 P$ to $1 S$ in actual measurement.

To examine the accuracy of the improved Deser formulas, we calculate the $K^{-} d$ scattering length by the fixed center approximation, which leads to $a_{K^{-} d}=(-1.42+i 1.60) \mathrm{fm}$. The estimated values of the shift and width by this value and Eqs. (1) and (2) are shown in Table 2. It is clear that the improved Deser formulas do not work accurately. The deviations from the exact value are of the order of $100 \mathrm{eV}$. This is consistent with the finding in the Faddeev approach [21].

One of the motivations to study kaonic deuterium is to provide further constraint on the $I=1$ component of the $\bar{K} N$ interaction. For this purpose, we vary the strength of the $\bar{K} N(I=$ 1) potential as

$$
\operatorname{Re} \hat{V}^{\bar{K} N(I=1)}(r) \rightarrow \beta\left[\operatorname{Re} \hat{V}^{\bar{K} N(I=1)}(r)\right]
$$


Table 2. Level shift and width of kaonic deuterium obtained by solving the three-body Schrödinger equation with the Kyoto $\bar{K} N$ potential, and by using the improved Deser formula (1) and its resummed version (2). The numerical data are taken from Ref. [19].

\begin{tabular}{lcc}
\hline & $\Delta E_{K^{-} d}(\mathrm{eV})$ & $\Gamma_{K^{-} d}(\mathrm{eV})$ \\
\hline Full Schrödinger equation & 670 & 1016 \\
Improved Deser formula (1) & 910 & 989 \\
Resummed formula (2) & 818 & 1188 \\
\hline
\end{tabular}

Table 3. Level shifts and decay widths of kaonic hydrogen and deuterium computed with different

$I=1$ strengths of the $\bar{K} N$ interaction. The experimental level shift data of kaonic hydrogen is $\left(\Delta E_{K^{-} p}, \Gamma_{K^{-} p}\right)=(283 \pm 36 \pm 6,541 \pm 89 \pm 22) \mathrm{eV}[9,10]$. The numerical data are taken from Ref. [19].

\begin{tabular}{rrrrr}
\hline$\beta$ & $\Delta E_{K^{-} p}(\mathrm{eV})$ & $\Gamma_{K^{-} p}(\mathrm{eV})$ & $\Delta E_{K^{-} d}(\mathrm{eV})$ & $\Gamma_{K^{-} d}(\mathrm{eV})$ \\
\hline 1.08 & 287 & 648 & 676 & 1020 \\
1.00 & 283 & 607 & 670 & 1016 \\
-0.17 & 310 & 430 & 506 & 980 \\
\hline
\end{tabular}

The parameter $\beta$ is constrained by the experimental uncertainty in the SIDDHARTA data of kaonic hydrogen $[9,10]$. Corresponding results for kaonic deuterium are summarized in Table 3. These results indicate that the measurement of kaonic deuterium with a $25 \%$ accuracy will improve the determination of the $I=1$ component of the $\bar{K} N$ interaction, in a more stringent manner than the existing kaonic hydrogen data.

\section{Conclusion}

We have studied kaonic deuterium with the accurate three-body calculation method combined with the realistic $\bar{K} N$ interactions. We conclude that

- the three-body calculation is mandatory to obtain the level shift and width of kaonic deuterium because the estimation by the improved Deser formulas is not accurate, and

- the properties of kaonic deuterium are sensitive to the $I=1$ component of the $\bar{K} N$ interaction.

Thus, we expect that the measurement of kaonic deuterium in the forthcoming experiments (J-PARC E15 and SIDDHARTA-2) will provide a clue to completely determine the isospin dependent $\bar{K} N$ interactions.

We are grateful to Tsubasa Hoshino and Shota Ohnishi for their significant contributions to the computation and discussions to complete this work. This work is in part supported by JSPS KAKENHI Grant Number JP16K17694 and by the Yukawa International Program for Quark-Hadron Sciences (YIPQS).

\section{References}

[1] A. Gal, E.V. Hungerford, D.J. Millener, Rev. Mod. Phys. 88, 035004 (2016)

[2] N. Kaiser, P.B. Siegel, W. Weise, Nucl. Phys. A 594, 325 (1995)

[3] E. Oset, A. Ramos, Nucl. Phys. A 635, 99 (1998)

[4] J.A. Oller, U.G. Meissner, Phys. Lett. B 500, 263 (2001)

[5] T. Hyodo, D. Jido, Prog. Part. Nucl. Phys. 67, 55 (2012)

[6] Y. Kamiya, K. Miyahara, S. Ohnishi, Y. Ikeda, T. Hyodo, E. Oset, W. Weise, Nucl. Phys. A 954, 41 (2016) 
[7] Y. Akaishi, T. Yamazaki, Phys. Rev. C 65, 044005 (2002)

[8] S. Ohnishi, W. Horiuchi, T. Hoshino, K. Miyahara, T. Hyodo, Phys. Rev. C 95, 065202 (2017)

[9] M. Bazzi, G. Beer, L. Bombelli, A. Bragadireanu, M. Cargnelli et al. (SIDDHARTA Collaboration), Phys. Lett. B 704, 113 (2011)

[10] M. Bazzi, G. Beer, L. Bombelli, A. Bragadireanu, M. Cargnelli et al. (SIDDHARTA Collaboration), Nucl. Phys. A 881, 88 (2012)

[11] Y. Ikeda, T. Hyodo, W. Weise, Phys. Lett. B 706, 63 (2011)

[12] Y. Ikeda, T. Hyodo, W. Weise, Nucl. Phys. A 881, 98 (2012)

[13] M. Tanabashi et al., Phys. Rev. D 98, 030001 (2018)

[14] Z.H. Guo, J.A. Oller, Phys. Rev. C 87, 035202 (2013)

[15] M. Mai, U.G. Meißner, Eur. Phys. J. A 51, 30 (2015)

[16] J. Zmeskal et al., Acta Phys. Polon. B 46, 101 (2015)

[17] C. Curceanu et al., Nucl. Phys. A 914, 251 (2013)

[18] M. Iliescu et al., J. Phys. Conf. Ser. 770, 012034 (2016)

[19] T. Hoshino, S. Ohnishi, W. Horiuchi, T. Hyodo, W. Weise, Phys. Rev. C 96, 045204 (2017)

[20] P. Doleschall, J. Revai, N.V. Shevchenko, Phys. Lett. B 744, 105 (2015)

[21] J. Revai, Phys. Rev. C 94, 054001 (2016)

[22] K. Varga, Y. Suzuki, Phys. Rev. C 52, 2885 (1995)

[23] Y. Suzuki, K. Varga, Lect. Notes Phys. Monogr. 54, 1 (1998)

[24] Y. Suzuki, W. Horiuchi, M. Orabi and K. Arai, Few Body Syst. 42, 33 (2008)

[25] J. Mitroy, S. Bubin, W. Horiuchi, Y. Suzuki, L. Adamowicz, W. Cencek, K. Szalewicz, J. Komasa, D. Blume, K. Varga, Rev. Mod. Phys. 85, 693 (2013)

[26] K. Miyahara, T. Hyodo, Phys. Rev. C 93, 015201 (2016)

[27] T. Hyodo, W. Weise, Phys. Rev. C 77, 035204 (2008)

[28] K. Miyahara, T. Hyodo, W. Weise, Phys. Rev. C 98, 025201 (2018)

[29] U.G. Meissner, U. Raha, A. Rusetsky, Eur. Phys. J. C 35, 349 (2004)

[30] U.G. Meissner, U. Raha, A. Rusetsky, Eur. Phys. J. C 47, 473 (2006)

[31] V. Baru, E. Epelbaum, A. Rusetsky, Eur. Phys. J. A 42, 111 (2009)

[32] D.R. Thompson, M. Lemere, Y.C. Tang, Nucl. Phys. A 286, 53 (1977) 